\title{
Neurotropic malignant melanoma of right temple with orbital metastasis: a clinicopathological case report*
}

\author{
M K KHALIL AND W P DUGUID \\ From the Departments of Pathology and Ophthalmology, Montreal General Hospital, McGill University, \\ Montreal, Quebec, Canada
}

SUMMARY A case is reported of neurotropic melanoma developed from a superficial spreading melanoma with minimal cytological deviation, situated in the right temple. The nine-year course was clinically characterised by local recurrences, involvement of the orbit and the parotid region via neurogenic invasion, and systemic metastases to lung, seventh rib, and the brain. The histopathology was characterised by fascicles of dysplastic spindle cells, neuroid arrangement in loose fibrillary matrix, and peri- and intraneural permeation of the nerve trunks. Despite minimal atypism the neoplasm metastasised and had a fatal outcome. The spindle cell component of the neurotropic melanoma lacked melanogenesis; Fontana stains were negative. As previously demonstrated, the histogenesis of the neurotropic melanoma is possibly a Schwann cell differentiation of the dysplastic atypical melanocytes, as shown by the positive reactions to Bodian's stain.

Neurotropic melanoma, a rare type of spindle cell malignant melanoma, is considered a variant of desmoplastic melanoma. ${ }^{12}$ Sutula et al. ${ }^{3}$ reported a case of desmoplastic melanoma of the upper eyelid in 1982. Reed and Leonard ${ }^{4}$ in 1979 reported 22 cases of neurotropic melanomas. Since then many other cases have been described..$^{-7}$ Our patient is one of the rare cases in which ocular tissue was involved during the nine-year course of a neurotropic malignant melanoma.

\section{Case report}

In May 1976 a 46-year-old man noticed that a longstanding freckle in the skin of the right temple had lately started to grow larger, and on occasions it bled when scratched. The pigmented lesion was biopsied and then totally excised by a plastic surgeon. The pathological diagnosis was 'melanose de Dubreuil'. Three years later, in November 1979, a deep small nodule appeared in the scar of the previous surgery.

\footnotetext{
*This study was presented in part at the annual meeting of the Eastern Ophthalmic Pathology Society, St Thomas, 1 November 1985.

Correspondence to M Khalil, MD, Department of Ophthalmology, 4th floor Livingston Hall, Montreal General Hospital, 1650 Cedar Avenue, Montreal, Quebec, Canada H3G 1A4
}

This was excised and found to be a 'recurrent malignant melanoma', which was incompletely excised. In January 1980 a fourth procedure was performed by a neurosurgeon, in which a wider field of excision was performed and part of the bony lateral orbital wall was removed, when specimens were taken from the orbital tissue. The pathological report was 'metastatic extension of melanoma into the right orbit'.

At this point an ophthalmologist was consulted. The vision was $20 / 20 \mathrm{OU}$ and the eye examination was unremarkable except for a slight limitation of mobility of the right eye on lateral and supralateral gaze and minimal ptosis and right frontalis muscle drop (eyebrow and eyelid) secondary to previous operations.

Extensive investigations revealed the absence of systemic metastases, and after numerous consultations a radical orbitectomy was performed followed by a local radiotherapy. The pathological examination showed intraneural malignant melanoma in the nerves situated in the fat of the lateral part of the orbit and in the area of the lacrimal gland. The orbital apex was free of tumour.

In July 1982 the patient suffered from severe right sided neuralgia, erythema, induration in the right parotid region, and right Bell's palsy. A biopsy from 


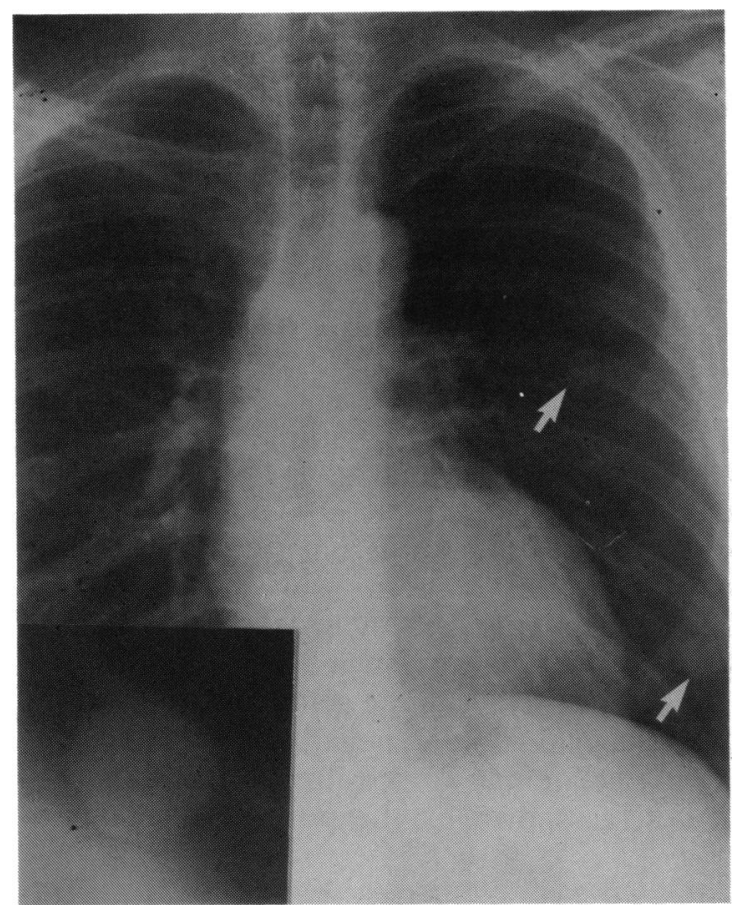

Fig. 1 October 1982. Chest $x$-ray, two metastatic nodules in left lung (arrows). Inset: Tomogram of the nodule in the base of the lung.

the parotid mass revealed a recurrence of the malignant melanoma along the ramifications of the facial nerve. Meanwhile, two metastatic nodules were seen in the chest $x$-ray (Fig. 1). A needle biopsy of one of the nodules proved it to be malignant melanoma metastasis. He was treated with irradiation to the

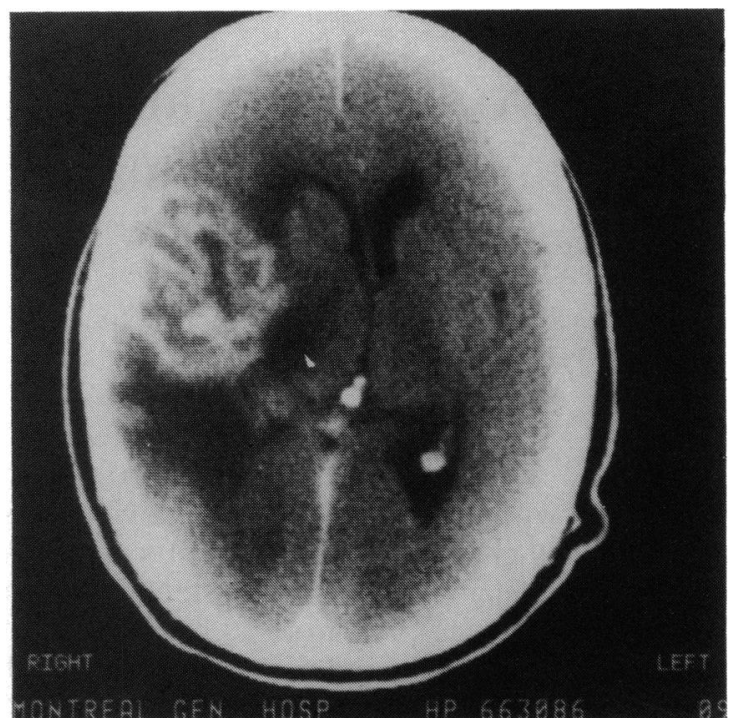

Fig. 2 February 1984. Brain CT scan, large necrotic mass in the parietofrontal region, considered to be metastasis.

parotid region, and chemotherapy was added after discovery of the lung metastases. Other possible metastases were detected by CT scans, in the seventh rib and in the right frontoparietal area of the brain (Fig. 2).

The patient's condition deteriorated progressively, and he died in early 1985 at the age of 55. No necropsy was performed.

\section{HISTOPATHOLOGY}

I. The primary skin lesion, biopsied and excised in 1976 , was found to be a superficial spreading malig-
Fig. 3 Histopathology of the precursory cutaneous melanotic lesion (1976), showing the features of superficial spreading melanoma. Atypical melanocytes were arranged as basilar nests in the elongated rete pegs. The cells were mostly spindled, a few naevoid and ballooned. (H and E).

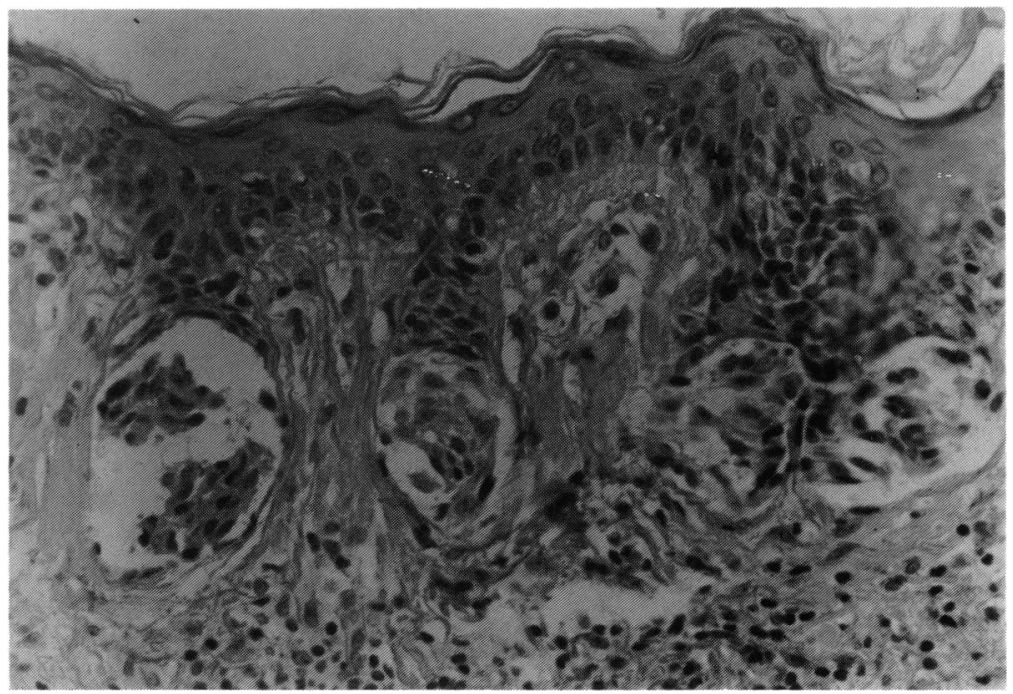




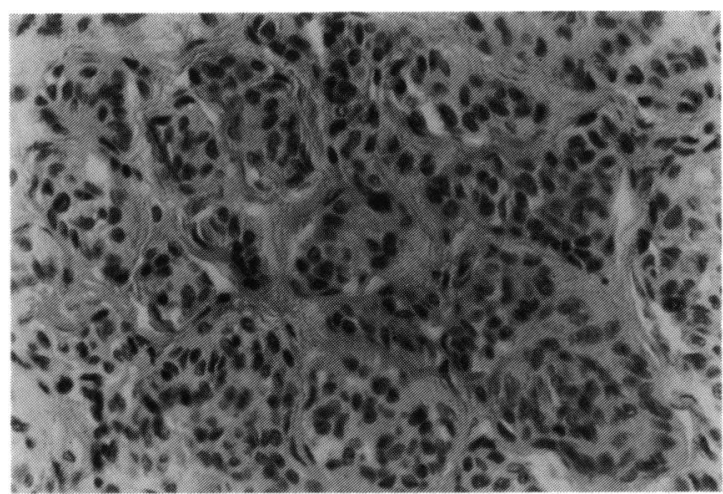

Fig. 4 A section from the central part of the skin lesion: aggregation of atypical melanocytes giving the overall configuration of a naevus; the cells were spindle shaped and naevoid in type with minimal atypia. ( $H$ and $E)$.

nant melanoma with 'minimal cytological deviation' and had the following features (Fig. 3). The pattern of proliferating atypical melanocytes was variablebasal nests, intraepithelial nests, and pagetoid and insitu-like patterns. The cell types of the atypical melanocytes were pleomorphic, small and large round, a few epithelioid, ballooned, and mainly spindled. The atypia was classified as mild, the divergence in nuclear size was minimal; some nuclei showed prominent nucleoli, and there was no mitosis. The subepithelial tissue (or dermis) showed

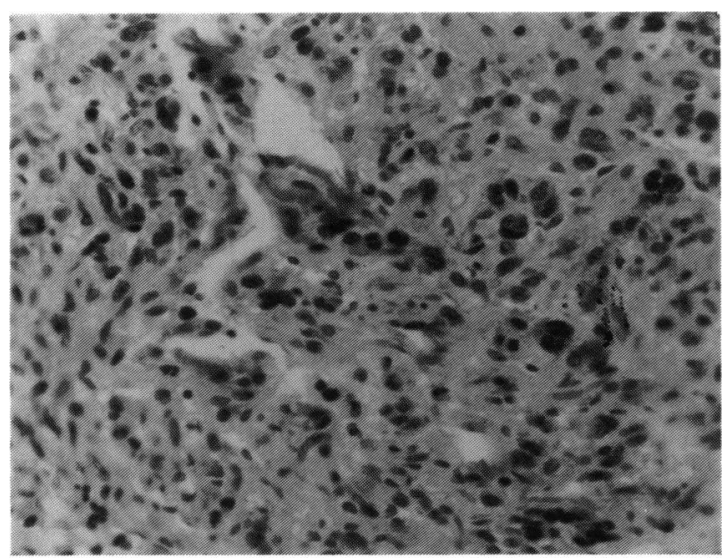

Fig. 5a

Fig. 5 Recurrent malignant melanoma of the skin (November 1979). (a) The cells were pleomorphic, spindle shaped, epithelioid, and multinucleated, and some showed mitotic figures. (b) Deep portion of the melanoma showing slender spindle cells arranged in fascicles in a loose fibrous matrix. The cells were aligned end to end. Their nuclei were elongated, hyperchromatic, different in size, and mitosis was not a prominent feature. (H and E). patchy mononuclear cell infiltration. The tumour cells extended deep in the dermis to the level of the sweat glands (1.4 $\mathrm{mm}$ in depth) and permeated the perineurium of the peripheral nerves at the deep edge of the dermis. In the centre of the lesion there was a nodule composed of aggregations of plump spindle cells which had the overall configuration consistent with a naevus, though more detailed study pointed to a 'minimal deviation melanoma' (Fig. 4).

II. The recurrent skin lesion which was incompletely removed in November 1979 and then underwent a wider excision in January 1980 showed the picture of 'a recurrent cutaneous malignant melanoma with intraneural invasion of the nerves'. In the superficial part of the mass the melanoma cells were pleomorphic (Fig. 5a)-naevoid, with a few epithelioid and multinucleated cells; the predominant cells were elongated plump spindle cells arranged in fascicles. The Fontana stain was weakly positive. The deeper portion of this lesion was composed of elongated slender spindle cells (Fig. 5b) with a neuroid arrangement in a background of loose fibrillary matrix. Some of the cells were aligned end to end. The cytoplasm of the cells was pale. The nuclei were oval and had densely uniform chromatin. Some nuclei showed margination of the chromatin and prominent nucleoli, indicating some degree of atypism, but mitotic figures were not evident. When the cells were cut horizontally, they appeared as small spindle cells. Intraneural invasion of many nerves with the same type of cells was seen. This portion of the tumour was negative for Fontana stain, but Bodian stain showed rigid silver-positive fibrils (axons) to be present.

III. The orbital biopsy obtained during the operative procedure of January 1980 showed amelanotic elongated spindle cells permeating the nerve trunks in the area of the lacrimal gland (Fig. 6). The cells had

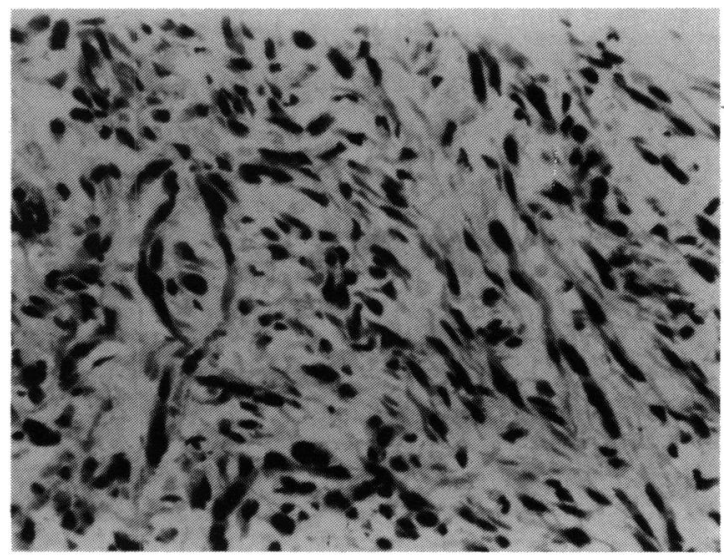

Fig. 5b 
Fig. 6 Orbital biopsy (January 1980). Lacrimal acini (on the right), the involvement of two nerves with tumour cells. Above: the normal trunk was surrounded by the tumour cells. Below: the tumour cells totally invaded and expanded the nerve. ( $H$ and $E$ ).

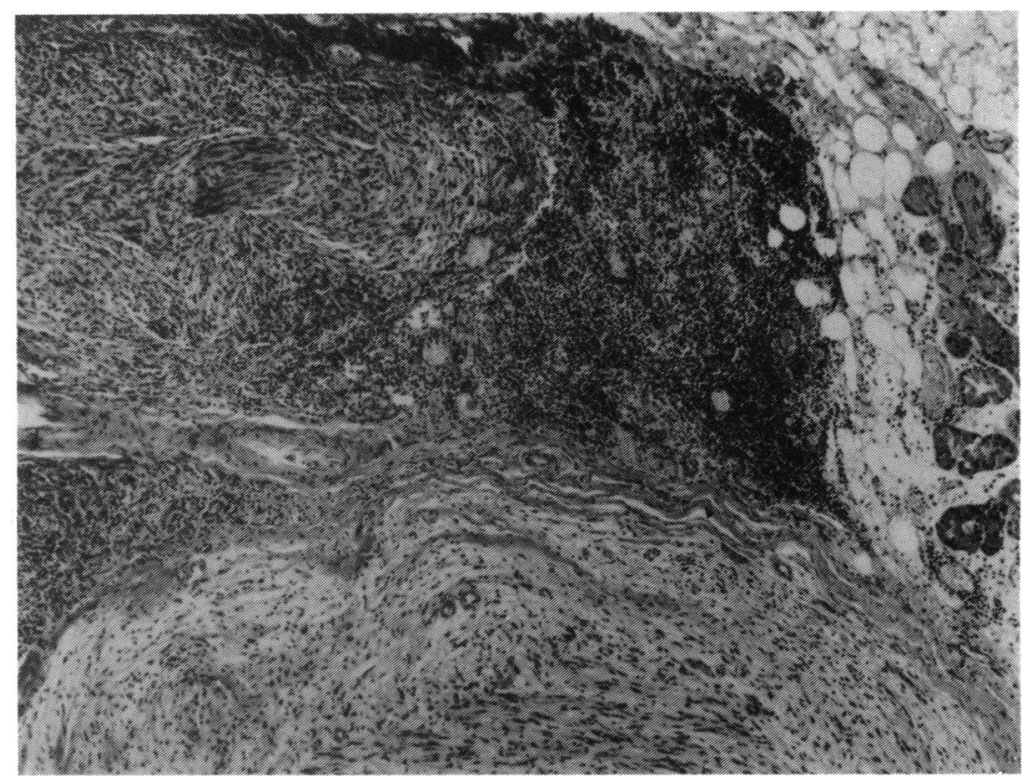

histopathological characteristics like those seen in the deep part of the skin lesion. The pathological diagnosis was 'orbital metastasis of a neurotropic malignant melanoma by peri- and intraneural extension'.

IV. In the specimen of the radical orbitectomy of April 1980 residual metastatic neurotropic malignant melanoma involved numerous orbital nerves in the area of the lacrimal gland in the lateral part of the orbit; the orbital apex was free of tumour. The histopathology was similar to that in the previous orbital biopsy, and the nerves were expanded with the same type of melanoma spindle cells. A mononuclear inflammatory cell reaction surrounded the involved nerves. Bodian's stain and S-100 protein immunoreactivity were positive.

V. The biopsy, which was obtained from the parotid region in July 1982, showed a neuroid arrangement of the same type of spindle cells. The melanoma cells were found to be intraneural and in the soft tissue outside the nerve sheaths. Fontana stains were negative, while Bodian's stain and S-100 protein immunoreactivity were positive. The diagnosis was 'metastatic extension of a neurotropic malignant melanoma along the ramifications of the facial nerve'.

The final clinicopathological diagnosis was: (1) neurotropic malignant melanoma arising on top of a superficial spreading melanoma with 'minimal cytological deviation'; (2) multiple metastases through neurogenic routes to the orbit and the parotid region; (3) systemic metastases to the lung, seventh rib, and possibly to the brain.

\section{Discussion}

Neurotropic melanoma, as recently described by Reed and Leonard, ${ }^{4}$ is a rare type of spindle cell malignant melanoma that invades the dermis from one of the following precursory epidermal lesions: (1) lentiginous melanoma, either a lentigo maligna or acral lentiginous melanoma; (2) a minimal deviation melanoma ${ }^{89}$ that is, a boderline lesion in which the histopathology mimics that of a benign naevus; (3) a de novo variety which lacks the evidence of a previous melanotic epidermal lesion.

The precursory lesion in our patient had the characteristic features of superficial spreading melanoma in its radial growth component, while its vertical growth phase was consistent with a minimal deviation melanoma. Dabbs and Bolen described a case of superficial spreading melanoma in which neurosarcomatous features were evident in the metastases. ${ }^{10}$

Most neurotropic melanomas occur on the exposed surfaces of the head and neck. In Reed and Leonard's series ${ }^{4}$ they developed primarily on the temple in three out of 22 patients. The orbit was not involved in any of the three cases, but two of them died with involvement of the central nervous system.

The histopathology of neurotropic melanoma has distinctive features. The pattern is characterised by, first, fasciculation: the dysplastic spindle cells of the epidermis invade the dermis in the form of fascicles of elongated slender spindle cells; and, secondly, neurotropism: the spindle cells have the affinity to permeate the peripheral nerves, and invasion of 
adjacent structures occurs via perineural and intraneural extensions.

A neuroid pattern tends to occur in soft tissues outside the nerve sheaths and in uncontrolled recurrent lesions. The picture appears as masses of spindle cells with neuroid arrangement. The neurotropic melanoma may be associated with a desmoplastic component, which leads to the production of bulky subcutaneous tumefactions.

As to cell type, these tumours are composed of slender, elongated, spindle cells usually aligned end to end. The cytoplasm is pale and the nuclei are oval and have dense chromatin. Some nuclei show margination of the chromatin and prominent nuclei. They lie in a background of loose fibrillary matrix rich in reticulin. In transverse sections cells appear as small spindle types.

In general the tumour cells show minimal atypism, but some nuclei are of different size and shape and contain prominent nuclei. Mitosis is scarce.

Despite the minimal features of atypism the behaviour of these tumours is aggressive and characterised by multiple recurrences, distant metastases, and fatal outcome. The neurogenic extension of the tumour cells poses great difficulty in effecting a complete local excision of these lesions and so total cure. In our patient a radical orbitectomy and wide excision of adjacent structures were performed, yet a neurogenic extension along the branches of the facial nerve resulted in a recurrent metastasis in the parotid region two years after surgery.

The orbital invasion from the primary skin lesion in the temple occurred by neurogenic spread either along nerve fibres connecting the first and second branches of the trigeminal nerve (V-I and V-II) though small canals in the lateral bony orbital wall or around the orbital rim following the branches of the lacrimal nerve.

Schwann cell differentiation has been suggested in the histogenesis of neurotropic melanomas. ${ }^{511}$ This was supported by certain observations made in light and electron microscopic studies of these tumours. The spindle cells are difficult to differentiate from Schwann cells and fibroblasts; they demonstrate marked affinity for cutaneous peripheral nerves and tend to occur in neuroid arrangements. Absence of melanogenesis: the spindle cells were amelanotic and Fontana stain was negative. In electron microscopic (EM) studies $^{510}$ melanosomes were absent. But Schwann cell features were identified on EM studies. ${ }^{10}$ Argyrophilic fibres that have the characteristic of axons were demonstrated in most cases with Bodian's stain.

The S-100 protein immunoreactivity test, although not specific for Schwann cells, is useful in differentiating desmoplastic and neurotropic malignant melanomas from other types of spindle cell tumour which cause diagnostic confusion (leiomyomas, spindle cell variant of squamous cell carcinoma, tumours of fibreoblastic origin, atypical fibroxanthoma, and adaptive fibroplasia). The only spindle cell tumours which stain positively with S-100 protein are neural tumours and spindle cell malignant melanomas. ${ }^{12}$ In our case the spindle cells in the deep part of the cutaneous recurrence of the malignant melanomas, the orbital extension, and the metastasis in the parotid region all stained with S-100 protein and were all amelanotic. Warner et al. " presented three cases of neurotropic malignant melanomas in which the spindle cells stained with S- 100 protein and melanosomes were absent on EM studies. S-100 protein-positive staining of the spindle cells in neurotropic malignant melanoma with the absence of melanogenesis is suggestive of the Schwannian nature of these cells. It is generally accepted that Schwann cells and melanocytes are derived from related precursors as neural crest derivatives. Schwann cells may become facultative melanocytes, and melanoblasts may assume Schwannian characteristics. Abundant morphological evidence illustrates the overlapping spectrum of differentiation among melanocytic and Schwannian lines. Such cases include the melanocytic schwannoma, pigmented neurofibroma, desmoplasmic malignant melanomas, and common cutaneous naevi.

Why there is a sudden transition from a melanocytic component to fascicles of Schwannian-spindle cells is still unclear. Many theories have been suggested. Cramer ${ }^{13}$ proposed the possibility that these tumour cells originate from the nerve-sheath precursor stage of the melanocytic differentiation pathway. The recurrences may reflect the persistence of neoplastic stem cells in the proximal nerve sheath. Reed and coworkers ${ }^{8}$ suggested that dermal melanocytes in contact with neurites transform into Schwann cells, melanogenesis is reduced, and fibrogenesis appears. Warner ${ }^{14}$ stated that the phenomenon of phenotypic mixing or conversion in neurotropic melanomas could be explained by cell hybridisation between dysplastic melanocytes and Schwann cells with variable expression.

\section{References}

1 Conley J, Lattes R, Orr W. Desmoplastic malignant melanoma (a rare variant of spindle cell melanoma). Cancer 1971; 28: 914-36.

2 Labrecque PG, Hu CH, Winkelmass RK. On the nature of desmoplastic melanoma. Cancer 1976; 38: 1205-13.

3 Sutula FC, Dortzbach RK, Balles JC. Desmoplastic malignant melanoma of the upper eyelid. Ann Ophthalmol 1982; 14: 141-3.

4 Reed RJ, Leonard DD. Neurotropic melanoma. A variant of desmoplastic melanoma. Am J Surg Pathol 1979; 3: 301-11. 
5 Warner TFCS, Hafez GR, Finch RE, Brandenberg JH. Schwann cell features in neurotropic melanoma. J Cutan Pathol 1981; 8: 177-87.

6 Ackerman AB, Godomski J. Neurotropic malignant melanoma and other neurotropic neoplasms in the skin. Am J Dermatol 1984; 6: 63-80.

7 Dupree WB. Case for diagnosis, neurotropic melanoma of neck. Milit Med 1985; 150: 165-7.

8 Reed RJ, Ichinose H, Clark WH Jr, Mihm MC Jr. Common and uncommon melanocytic nevi and borderline melanomas. Semin Oncol 1975; 2: 119-47.

9 Warner TFCS, Seo IS, Bennett JE. Minimal deviation melanoma with epidermtropic metastases arising in a congenital nevus. Am J Surg Pathol 1980; 4: 175-83.

10 Dabbs DJ, Bolen JW. Superficial spreading malignant melanoma with neurosarcomatous metastasis. Am J Clin Pathol 1984; 82: 109-14.

11 Warner TFCS, Lloyd RV, Hafez GR, Angerine JM. Immunochemistry of neurotropic melanoma. Cancer 1984; 53: 254-7.

12 From L, Hanna W, Kahn HJ, Gruss J, Marks A, Baumal R. Origin of the desmoplasia in desmoplastic malignant melanoma. Hum Pathol 1983; 14: 1072-80.

13 Cramer SF. The neoplastic development of malignant melanoma. A biological rationale. Am J Dermatopathal 1984; 6: 299-308.

14 Warner TFCS, Cell hybridization: an explanation for phenotypic diversity of certain tumours. Med Hypotheses 1975; 1: 51-7.

Accepted for publication 14 April 1986. 